\title{
Wavy Taylor vortices in molecular dynamics simulation of cylindrical Couette flow
}

\author{
David J. Trevelyan ${ }^{1}$ and Tamer A. Zaki ${ }^{1,2, *}$ \\ ${ }^{1}$ Department of Mechanical Engineering, Imperial College London, Exhibition Road, London, SW7 2AZ, United Kingdom \\ ${ }^{2}$ Department of Mechanical Engineering, Johns Hopkins University, Baltimore, Maryland 21218, USA
}

(Received 2 October 2015; published 5 April 2016)

\begin{abstract}
Molecular dynamics simulations of flow between concentric rotating cylinders are performed. As the relative speed between the two cylinders is increased, a spontaneous flow bifurcation occurs and vortices form in a stationary-vortex or traveling-wavy-vortex configuration. The former emerges when the axial boundary conditions constrain the flow by reflection, and the traveling-wavy-vortex flow develops when the axial boundaries are relaxed to periodic conditions. The flow bifurcation is triggered by the thermal fluctuations in the system, and the resulting flow field is in agreement with previous experimental observations. In addition, the temporal growth of the Fourier mode that characterizes the wavy-vortex motion is well described by Landau's theory for Hopf bifurcations. The spatiotemporal energy spectrum is evaluated in order to characterize the instability in terms of its azimuthal wave number and wave speed.
\end{abstract}

DOI: 10.1103/PhysRevE.93.043107

\section{INTRODUCTION}

Where accurate continuum models of complex flow phenomena are unavailable, molecular dynamics simulations have provided an alternative. Examples include the moving contact line problem [1], complex fluid rheology [2], and slip at a solid-liquid interface [3]. Common among these examples is a sensitivity of the macroscopic flow dynamics to phenomena that occur at molecular length and time scales. Another area of both fundamental and practical importance is the onset of hydrodynamic instability, and whether flow bifurcation can take place due to thermal fluctuations associated with random molecular motions, rather than additional external forcing. Applications span a wide range of scales from the onset of elastic chaos in polymer melts [4] to transition to turbulence in aerospace vehicles [5]. When a flow parameter is increased beyond some critical value, a primary instability causes the basic state to bifurcate and a new flow pattern emerges. Secondary, and potentially tertiary, flow instabilities are also possible, which can lead to subsequent bifurcations.

In order to accurately probe an inherent flow instability, it is important to examine the dynamics of the flow in the absence of any additional sources of noise beyond the thermal fluctuations in the system. While this is a challenging task in laboratory experiments, numerical simulations can achieve this objective precisely by eliminating any source of external noise. In addition, molecular dynamics simulations inherently capture thermal noise in a system by explicitly modeling molecular motion. Previous molecular dynamics simulations have reproduced the primary instabilities that lead to Rayleigh-Bénard convection [6,7], vortex shedding [8,9], and Taylor-Couette flow [10-12]. In this work, the focus is on the onset of the secondary instability of the flow between rotating concentric cylinders, and whether it can be accurately captured using molecular dynamics simulations.

The base state of interest is cylindrical Couette flow, which develops between two concentric rotating cylinders. A

\footnotetext{
*t.zaki@jhu.edu
}

schematic of the geometry is provided in Fig. 1. For a Newtonian fluid, the nondimensional parameter that determines the stability of the base state is the Taylor number,

$$
\mathcal{T} \equiv 4 \mathcal{R}^{2} \frac{1-\eta}{1+\eta}
$$

where $\eta=R_{i} / R_{o}$ is the ratio of inner- to outer-cylinder radii, and $\mathcal{R}$ is the Reynolds number. With a fixed outer cylinder, the flow stability is dictated entirely by the Reynolds number,

$$
\mathcal{R} \equiv \frac{\rho R_{i} \Omega_{i} d}{\mu},
$$

where $\Omega_{i}$ is the angular velocity of the inner cylinder, $d=$ $R_{o}-R_{i}$ is the gap width, $\rho$ is the fluid density, and $\mu$ is its shear viscosity. As the Reynolds number is increased beyond the first critical value, $\mathcal{R}_{c}$, toroidal Taylor vortices emerge superposed onto the basic cylindrical Couette flow. This flow configuration is referred to as Taylor-Couette flow. Streamlines in this flow orbit the vortex centers in the $r-z$ plane, and the velocity field is axisymmetric in the azimuthal coordinate, $\theta$. The flow becomes unstable again, via a Hopf bifurcation, at a second critical Reynolds number $\mathcal{R}_{c}^{\prime}>\mathcal{R}_{c}$. At this point, a waviness develops and the new state is described as a "wavyvortex" flow. The waves are convected around the azimuth at a constant speed that is approximately linear in $\Omega_{i}$ [13], and the streamlines are no longer confined to a single vortex [14]. Progressively increasing the Reynolds number even further results in a series of new bifurcations which eventually lead to a fully turbulent regime.

Taylor's seminal work [15] provided a linear stability analysis of the base flow and predicted the criterion for the primary macroscopic instability. The assumption here is that the bifurcation can be triggered by infinitesimally small perturbations, naturally present in any experiment. Whether molecular thermal fluctuations are sufficient to trigger the flow bifurcation is not incorporated into the analysis. Beyond the primary instability, subsequent bifurcations have been the subject of a considerable body of work in experiments [13,1619], theory [20-23], and continuum simulations [24-28]. However, very few studies of Taylor-Couette flow have been reported in which the fluid is treated with a molecular model. 


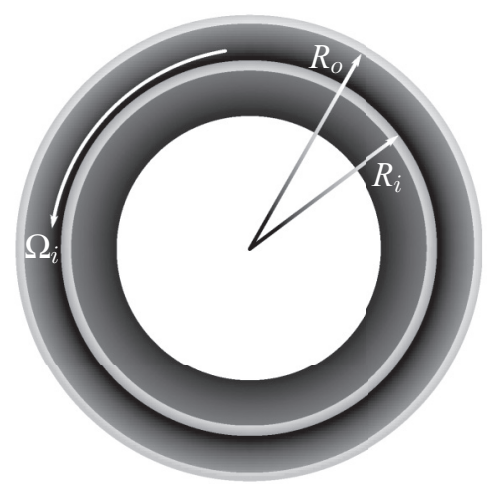

FIG. 1. A fluid, bounded by solid cylinders, occupies the region $R_{i}<r<R_{o}$, where $r$ is the radial coordinate. The outer cylinder remains at rest while the inner cylinder rotates with angular velocity $\Omega_{i}$.

Hirshfeld and Rapaport [10-12] have presented the first and only studies to date that have demonstrated the appearance of the Taylor-Couette instability in a molecular dynamics simulation. They found excellent quantitative agreement between their evaluation of the critical Taylor number and those of previous experiments. To save computational expense, they chose to simulate only a quarter of the azimuthal domain by adopting periodic boundary conditions. However, by only considering a quarter of the full cylinder, they restricted any possible azimuthal instabilities to those with integer wave numbers $k_{\theta}$ that are multiples of 4 . There have also been a small number of direct simulation Monte Carlo studies of instabilities in Taylor-Couette flow [29-31], but none have given rise to the wavy-vortex pattern spontaneously.

The present work examines whether molecular dynamics simulations can accurately reproduce the development of the wavy-vortex regime, without artificially exciting or directly imposing a wavy initial flow field. The effect of the axial boundary condition on the appearance of the instability is explored, and any temporal growth of the wave amplitude is compared to Landau's model [32] of hydrodynamic stability. Analysis is provided of the full three-dimensional velocity fields alongside the key spatiotemporal spectra of the flow.

The remainder of this paper is organized as follows. In Sec. II, details of the simulation setup are provided, along with the procedure for the two types of axial boundary conditions considered herein. The results are reported in Sec. III, where the velocity fields are presented first, followed by a discussion of the spectra. Finally, a summary of the findings is provided in Sec. IV.

\section{SIMULATION DETAILS}

The fluid considered in this work consists of soft spheres that interact with the repulsive part of the Lennard-Jones potential $\phi_{j k}$,

$$
\phi_{j k}= \begin{cases}4 \epsilon\left[\left(\frac{\sigma}{\tilde{q}_{j k}}\right)^{12}-\left(\frac{\sigma}{\tilde{q}_{j k}}\right)^{6}\right]+\epsilon, & \tilde{q}_{j k}<2^{1 / 6} \sigma \\ 0, & \tilde{q}_{j k} \geqslant 2^{1 / 6} \sigma\end{cases}
$$

where $\tilde{q}_{j k}=\left|\tilde{\mathbf{q}}_{k}-\tilde{\mathbf{q}}_{j}\right|$ is the magnitude of the vector, $\tilde{\mathbf{q}}_{j k}$, that separates the positions of particles $j$ and $k$. Reduced units are adopted throughout, for which $\sigma=\epsilon=1$. The fluid region, $R_{i}<r<R_{o}$, is bounded in the radial coordinate, $r$, by cylindrical walls. Both cylinder walls consist of soft spheres that, while identical to the fluid particles in both size and mass, experience an additional tethering force to lattice sites. The standard molecular dynamics equations of motion [33] are integrated in a Cartesian coordinate system, for which position and momentum are denoted by $\tilde{\mathbf{q}} \equiv(x, y, z)$ and $\tilde{\mathbf{p}} \equiv\left(p_{x}, p_{y}, p_{z}\right)$, respectively. To extract useful statistics, these variables are also expressed in cylindrical coordinates as $\mathbf{q} \equiv(r, \theta, z)$ and $\mathbf{p} \equiv\left(p_{r}, p_{\theta}, p_{z}\right)$. The initialization of the system and the flow simulation are performed in stages, which are discussed below.

\section{A. Simulation initialization}

The initialization procedure is performed in two stages. First, the cylindrical walls are constructed. Second, the region between the walls is filled with fluid molecules and the system is allowed to equilibrate at rest.

The cylinder walls are formed by constructing a large fcc lattice, at density $\rho_{c}=1.0$, and particles that are not part of the cylinder walls are removed. Figure 1 shows a schematic of the cylinder geometry viewed along the $z$ axis. The cylinder radii are $R_{i}=399$ and $R_{o}=457$, and the domain length is $L_{z}=451$. The ratio of radii and aspect ratio are, therefore, $\eta=0.873$ and $\Gamma \equiv L_{z} / d=7.78$, respectively.

During the initialization of the cylinder walls, all particles interact with only the soft-sphere potential given by Eq. (3). In order to ensure that the surfaces of the cylinders are not unphysically rough, the particles are allowed to equilibrate between specularly reflecting radial boundaries. The leapfrog algorithm is chosen for integration, with time step $\Delta t=0.005$, and the initial thermal velocities are set so that the average kinetic temperature of the system is $T=0.2$. No further temperature controls are applied at this stage. Inspection of the final wall configuration reveals a system of close-packed planes that are smooth and curved normal to the radial axis.

The final particle positions are chosen as initial equilibrium sites, $\tilde{\mathbf{q}}_{j}^{\text {eq. }}$, to which the cylinder particles are then tethered via an additional anharmonic spring potential from the work by Petravic and Harrowell [34],

$$
\phi_{\mathrm{anh}}\left(\tilde{\mathbf{q}}_{j}\right)=-\kappa_{4}\left|\tilde{\mathbf{q}}_{j}-\tilde{\mathbf{q}}_{j}^{\mathrm{eq}}\right|^{4}-\kappa_{6}\left|\tilde{\mathbf{q}}_{j}-\tilde{\mathbf{q}}_{j}^{\mathrm{eq}}\right|^{6}
$$

where $\tilde{\mathbf{q}}_{j}-\tilde{\mathbf{q}}_{j}^{\text {eq }}$ is the separation vector between particle $j$ and its equilibrium position, $\kappa_{4}=5 \times 10^{3}$ and $\kappa_{6}=5 \times 10^{6}$.

The gap between the two cylinders, $R_{i}<r<R_{o}$, is filled with fluid molecules with bulk density $\rho_{f}=0.5$. The adopted fluid potential is given by Eq. (3). During this stage of the simulation setup, domain boundaries in the axial $(z)$ direction are specularly reflecting walls, and the fluid is confined in the radial $(r)$ direction by the tethered cylinder particles. The total number of particles in the system is 39 million.

\section{B. Simulation procedure}

After initialization, the simulation is performed in two stages that differ according to the choice of boundary condition 
in the axial $(z)$ coordinate. During the first stage, the angular velocity of the inner cylinder is gradually increased to the target speed and the velocity field is allowed to reach a steady state. Throughout this period the axial boundary condition is perfect slip, which is established by specular reflection. After the velocity field reaches a steady state, the specular end walls are removed and replaced by periodic boundaries. The simulation is then continued.

The rotation of the inner cylinder is performed by incrementing the azimuthal equilibrium coordinates of the solid particles in time,

$$
\mathbf{q}_{j}^{\mathrm{eq}}(t):=\mathbf{q}_{j}^{\mathrm{eq}}(t=0)+\int_{0}^{t} \dot{\theta}(t) r_{j} d t \mathbf{e}_{\theta} .
$$

Here, $\mathbf{e}_{\theta}$ is the unit vector in the $\theta$ direction, and $\dot{\theta}_{i}(t)$ is the angular velocity of the inner cylinder. The rotation speed is increased smoothly from $t=0$ according to a cosine envelope function,

$$
\dot{\theta}_{i}(t)= \begin{cases}\frac{1}{2}\left[1-\cos \left(\pi \frac{t}{t^{\prime}}\right)\right] \Omega_{i}, & 0 \leqslant t \leqslant t^{\prime}, \\ \Omega_{i}, & t>t^{\prime}\end{cases}
$$

where $\Omega_{i}=0.0124$ is the target angular speed, and the rampup time, $t^{\prime}=500$, is set to be similar to the time required for a single complete rotation of the inner cylinder at its maximum speed. The flow field is also allowed to develop for a further $t^{\prime \prime}=500$ time units before statistical sampling. To ensure that flow instabilities are neither triggered nor damped by artifacts of a thermostat algorithm, the temperature of only the cylinder wall particles is controlled, using the standard Nosé-Hoover thermostat. The inner- and outer-wall temperatures are set to $T_{i}=T_{o}=1.0$.

For $\eta=0.873$, the first critical Reynolds number is predicted by theory to be $\mathcal{R}_{c}=117.3$; see, e.g., [23]. The experiments by Coles [16] and Andereck, Liu, and Swinney [19] indicate that wavy-vortex flow is expected to appear in a similar geometry at $\mathcal{R}_{c}^{\prime} \approx 1.25 \mathcal{R}_{c}$. The Reynolds number in the present study is not known until the viscosity is evaluated from the simulation; however the results in the following section indicate that $\mathcal{R} \approx 163$.

\section{RESULTS AND DISCUSSION}

The results in this section compare the flow fields that develop with (a) reflective and (b) periodic axial boundary conditions. Field variables are coarse-grained by averaging particle velocities and forces within $25 \times 80 \times 96$ subvolumes in the $r, \theta$, and $z$ directions, respectively. A single sample is taken by averaging 20 microstates that are separated by 0.125 time units, which is sufficient time for the molecular configurations to become decorrelated [35]. The outcome is an averaged quantity over an interval spanning $\Delta \tau=2.5$ time units. The resulting time series is representative of the fluid streaming velocity field $\mathbf{u}(r, \theta, z, \tau) \equiv\left(u_{r}, u_{\theta}, u_{z}\right)$, where the new time coordinate, $\tau=t-\left(t^{\prime}+t^{\prime \prime}\right)$, represents the time from the start of statistical sampling.

The streaming velocity field is observed to have reached a steady state by $\tau=6000$. The mean azimuthal velocity in the

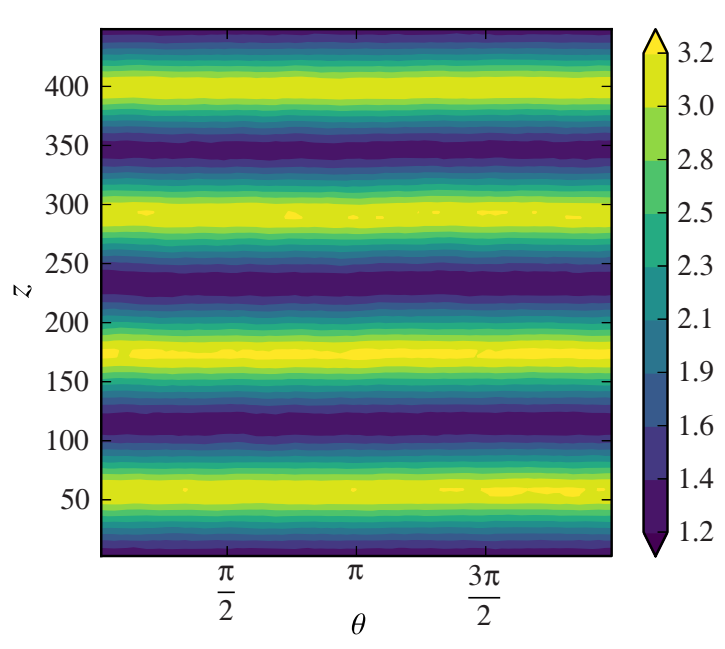

FIG. 2. Contours of $\left\langle u_{\theta}\right\rangle_{r, \tau}$ [see Eq. (7)] showing the flow field resulting from specularly reflecting end wall boundary conditions. The average is taken over an interval of $L_{\tau}=62.5$ time units, centered at $\tau=6000$.

$(\theta, z)$ plane is shown in Fig. 2, and is evaluated by

$$
\left\langle u_{\theta}\right\rangle_{r, \tau}=\frac{1}{L_{\tau} d} \int_{\tau_{-}}^{\tau_{+}} \int_{R_{i}}^{R_{o}} u_{\theta}(r, \theta, z, \tau) d r d \tau,
$$

where angle brackets $\langle\ldots\rangle_{X}$ hereafter represent an average over the $X$ coordinate(s). In this case the average is performed over the radial coordinate and the time interval $L_{\tau}=\tau_{+}$ $\tau_{-}$, where the bounds, $\tau_{ \pm}=\tau \pm L_{\tau} / 2$, are equidistant from a sample time, $\tau$. The existence of the stationary Taylor vortex is clear when the contours in Fig. 2 are compared with the streamline plots in Fig. 3.

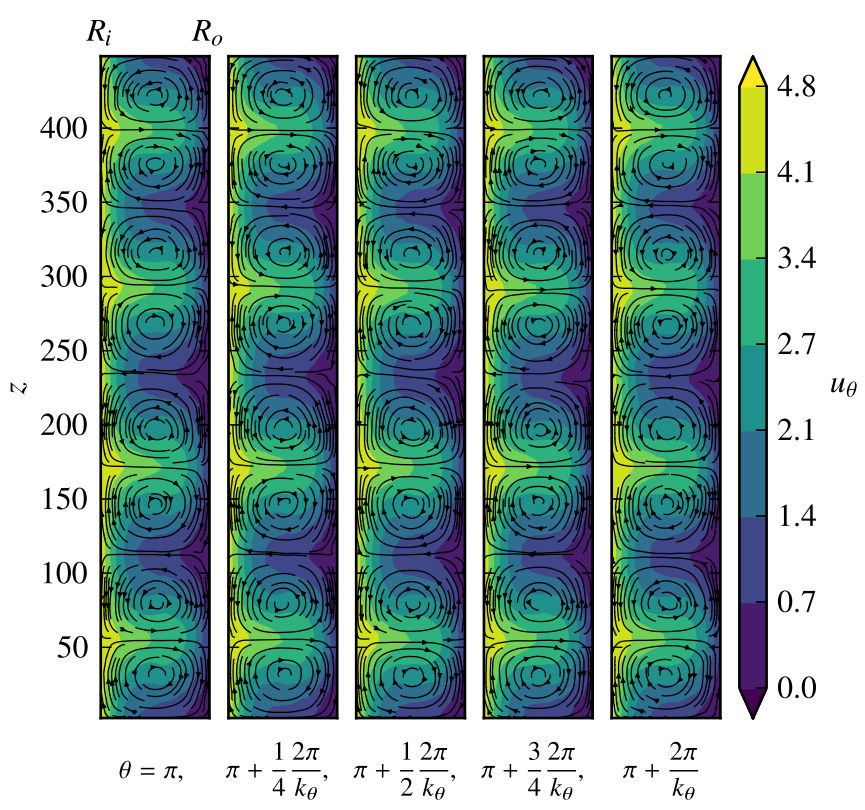

FIG. 3. Streamlines in the $r-z$ plane at 5 values of $\theta$, spread across a total angle $2 \pi / k_{\theta}$, where $k_{\theta}=5$. Streamlines are plotted over contours of $u_{\theta}(r, z)$. Averages are taken over an interval of $L_{\tau}=$ 37.5 time units, centered at $\tau=6000$, and over an azimuthal range $L_{\theta}=\pi / 20$ radians. 
Regions of fast-flowing fluid correspond to outward ejection of high-momentum fluid from the vicinity of the rotating inner cylinder towards the stationary outer cylinder. Conversely, regions of slower-moving fluid coincide with displacement of low-momentum fluid from the outer cylinder towards the inner one. The Taylor vortex is azimuthally invariant, i.e., $\partial \mathbf{u} / \partial \theta=0$, and similar results have been observed in previous molecular dynamics simulations [10-12] that employ reflecting end wall axial boundary conditions.

The Reynolds number is estimated here as

$$
\mathcal{R}=\lim _{r \rightarrow R_{i}^{+}}\left\langle\frac{\langle\rho\rangle_{z, \tau}\left\langle u_{\theta}\right\rangle_{z, \tau} d}{\langle\mu\rangle_{z, \tau}}\right\rangle_{\theta},
$$

where the viscosity is recovered from

$$
\langle\mu\rangle_{z, \tau}=\frac{\left\langle\zeta_{r \theta}\right\rangle_{z, \tau}}{\left\langle\left[\nabla \mathbf{u}+(\nabla \mathbf{u})^{T}\right]_{r \theta}\right\rangle_{z, \tau}} .
$$

In the equation above, $\varsigma_{r \theta}$ is the appropriate shear component of the stress tensor $\boldsymbol{S}$ (an expression for $\boldsymbol{S}$ in cylindrical coordinates is provided in the appendix). The corresponding $r-\theta$ component of the rate-of-strain tensor is given by

$$
\left[\nabla \mathbf{u}+(\nabla \mathbf{u})^{T}\right]_{r \theta} \equiv \frac{\partial u_{\theta}}{\partial r}-\frac{u_{\theta}}{r}+\frac{1}{r} \frac{\partial u_{r}}{\partial \theta},
$$

which is evaluated using a central-difference approximation. The estimated value of the Reynolds number is $\mathcal{R}=163$. This value is approximately $15 \%$ above the second critical Reynolds number, $\mathcal{R}_{c}^{\prime} \approx 140$, at which the wavy vortex has been observed to emerge in experiments with similar geometric configuration [19]. It is clear from Figs. 2 and 3 that, for the case with reflecting end wall boundary conditions, no waviness has developed on the Taylor vortices in the present simulations.

Upon replacing the reflective end walls at $\tau=6000$ with periodic boundaries, the flow field developed into a wavyvortex configuration. The mean azimuthal velocity in the $\theta-z$ plane, $\left\langle u_{\theta}\right\rangle_{r, \tau}$, is shown in Fig. 4, where a wavy mode with azimuthal wave number $k_{\theta}=5$ is observed. Similarly to the Taylor-vortex configuration, the regions of faster azimuthal flow coincide with radially outward advection, and regions of lower momentum coincide with inward advection. However, the extrema in $u_{\theta}$ for the wavy case now appear at the wave maxima and minima, rather than homogeneously through the centers of the vortices. The streamlines in Fig. 5 show that an additional mixing between the vortices, a phenomenon that is also observed in experiments [14], is another feature of the wavy-vortex flow that is absent from the pure Taylor vortex. The two states are also differentiated by the radial positions of the vortex centers. The centers of the Taylor vortices are aligned in $r$, whereas the radial locations of the centers of the wavy vortices meander. Despite this difference, the vortex centers in both cases are located at positions where the tangential velocity is approximately $u_{\theta} \approx 0.4 R_{i} \Omega_{i}$. This behavior is again consistent with the experimental observations by Akonur and Lueptow [14].

Attention is now directed to the spectral content of the flow field. Since eight vortices are formed along the axial direction, it is expected that Fourier analysis yields a peak in the energy spectrum at the fourth axial wave number. This peak will be

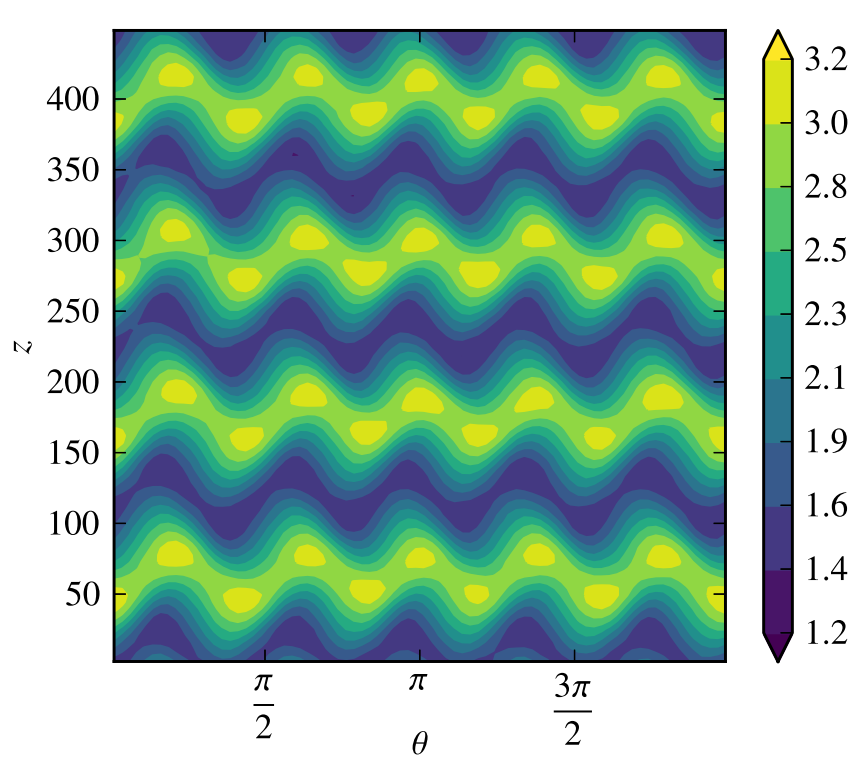

FIG. 4. Contours of $\left\langle u_{\theta}\right\rangle_{r}$ [see Eq. (7)] showing the flow field resulting from periodic axial boundary conditions. The average is taken over an interval of $L_{\tau}=62.5$ time units, centered at $\tau=20500$.

used to characterize the strength of the Taylor-vortex motion. In addition, in the wavy-vortex case, a peak in the energy spectrum is anticipated at the fifth azimuthal wave number which reflects the presence of five waves in that dimension. This peak will be used to characterize the strength of the wavy vortex.

The two-dimensional energy spectrum is evaluated as follows. First, the time series of the radial velocity, $u_{r}$, is

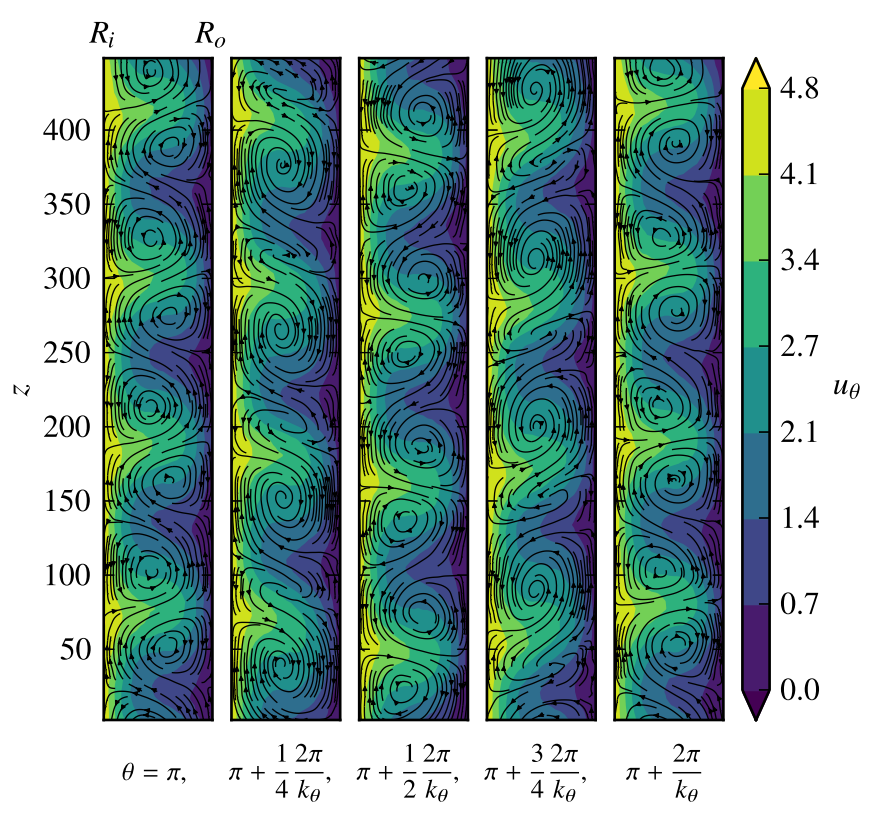

FIG. 5. Streamlines in the $r-z$ plane at 5 values of $\theta$, spread across a total angle $2 \pi / k_{\theta}$, such that a single oscillation of a vortex wave $\left(k_{\theta}=5\right)$ is enclosed. Streamlines are plotted over contours of $u_{\theta}(r, z)$. Averages are taken over an interval of $L_{\tau}=37.5$ time units, centered at $\tau=20500$, and over an azimuthal range $L_{\theta}=\pi / 20$ radians. 


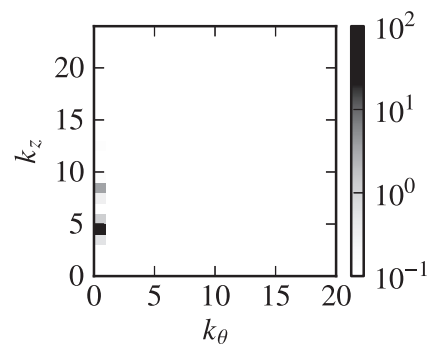

(a)

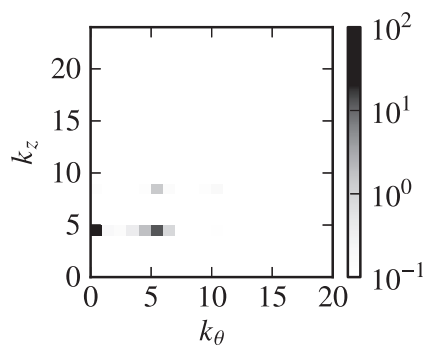

(b)
FIG. 6. Time-averaged energy spectra $\beta E_{r}\left(k_{\theta}, k_{z}\right)$ [see Eqs. (12) and (13)] for (a) reflecting end walls and (b) periodic axial boundary conditions. The time series is sampled for $L_{\tau}=25$ time units, centered at (a) $\tau=6000$ and (b) $\tau=20500$.

averaged in the radial direction,

$$
\left\langle u_{r}\right\rangle_{r}=\frac{1}{d} \int_{R_{i}}^{R_{o}} u_{r}(r, \theta, z, \tau) d r
$$

The $\left\langle u_{r}\right\rangle_{r}$ signal is then Fourier-transformed along the $z$ and $\theta$ coordinates,

$$
\hat{u}_{r}\left(k_{\theta}, k_{z}, \tau\right)=\frac{1}{2 \pi L_{z}} \int_{0}^{2 \pi} \int_{0}^{L_{z}}\left\langle u_{r}\right\rangle_{r} e^{-i\left(k_{\theta} \theta+2 \pi \frac{k_{z} z}{L_{z}}\right)} d z d \theta,
$$

where $k_{\theta}$ and $k_{z}$ are the integer wave numbers in the $\theta$ and $z$ directions, respectively. The average energy in each wave number is then taken over a sampling interval $L_{\tau}$,

$$
E_{r}\left(k_{\theta}, k_{z}\right)=\frac{1}{L_{\tau}} \int_{\tau_{-}}^{\tau_{+}}\left|\hat{u}_{r}\left(k_{\theta}, k_{z}, \tau\right)\right|^{2} d \tau,
$$

where $\tau_{ \pm}=\tau \pm L_{\tau} / 2$, and the subscript on $E_{r}$ denotes that the energy was computed from the radial component of velocity, $u_{r}$.

A comparison of the resulting energy spectra $\beta E_{r}\left(k_{\theta}, k_{z}\right)$ for both types of axial boundary conditions is shown in Fig. 6 . The energy in both plots has been normalized by the thermal energy scale $\beta^{-1}=k_{B} T$. The first component of interest is the peak at $E_{r}(0,4)$. This corresponds to the 8 vortices along the axial extent of the cylinders, and is the fundamental component of the Taylor vortex. Because velocities within the Taylor vortex reach high amplitude, nonlinear energy transfer injects energy into $E_{r}(0,8)$ and higher harmonics. The wavy-vortex motion is characterized by the peak at $E_{r}(5,4)$, which only appears in case (b) with periodic boundary conditions. During the bifurcation to the wavy-vortex state, energy is transferred from the azimuthally symmetric mode, $E_{r}(0,4)$, to $E_{r}(5,4)$. The magnitude of the axial harmonic at $E_{r}(0,8)$, resulting from the saturation of $E_{r}(0,4)$, is therefore reduced. The nonlinear interaction between the $E_{r}(0,4)$ and $E_{r}(5,4)$ modes also injects energy into $E_{r}(5,8)$. This mode is observed to be stronger than $E_{r}(0,8)$ in the spectrum of the wavy vortex.

The time evolution of the peaks in the energy spectrum for the Taylor- and wavy-vortices is presented in Fig. 7, where each sample time is $L_{\tau}=25$ time units. The top panel shows the evolution of the energy in the Taylor-vortex mode, and the bottom half shows the equivalent energy in the wavy-vortex mode. For times $\tau \leqslant 6000$, the axial boundary conditions are provided by reflecting end walls, and solid gray lines
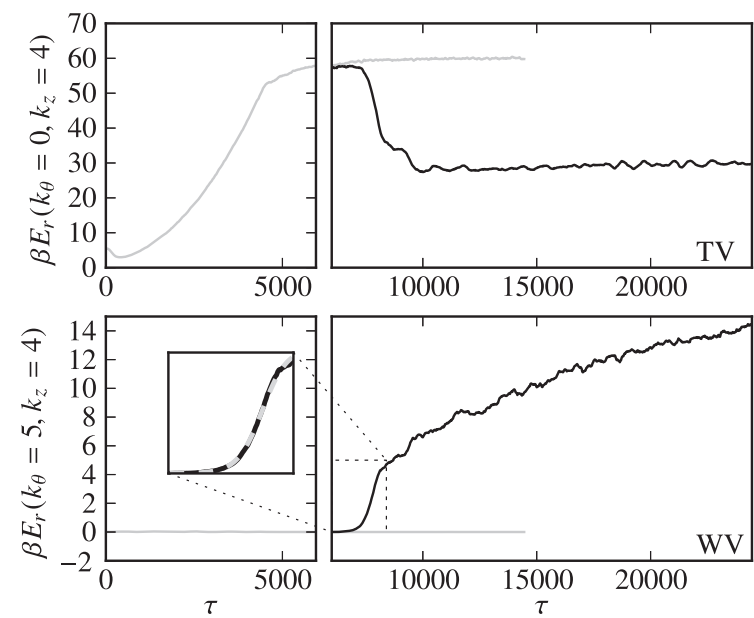

FIG. 7. Time evolution of spectral amplitudes $\beta E_{r}\left(k_{\theta}, k_{z}\right)$ characteristic of Taylor-vortex (TV, upper axes) and wavy-vortex (WV, lower axes) flow configurations. Solid gray lines correspond to end wall boundary conditions, and black lines to periodic boundaries. The gray dashed line in the inset shows a least-squares fit to Eq. (15).

in the figure show the energies for this configuration. Solid black lines mark the energies for the stage of the simulation where the end walls are removed and replaced by periodic boundaries. For an extra comparison, the results from an additional simulation in which the end walls are not removed for $\tau>6000$ are also plotted as a continuation of the solid gray lines. The energy in the wavy vortex remains zero in this case and the energy in the Taylor vortex remains constant. In the case where periodic axial boundary conditions are introduced, the energy in the wavy-vortex mode begins to grow almost immediately after $\tau=6000$. At the onset of the instability, the amplitude of the wavy-vortex peak is compared to the transient dynamics of a Hopf bifurcation. The temporal variation in oscillation amplitude $A(t)$ of a Hopf bifurcation is governed by the Landau amplitude equation,

$$
\tau_{0} \frac{d A}{d t}=\alpha A-\frac{A^{3}}{A_{0}^{2}},
$$

where $\tau_{0}$ and $A_{0}$ are scaling factors for time and amplitude, respectively, and the parameter $\alpha=\left(\mathcal{R}-\mathcal{R}^{\prime}{ }_{c}\right) / \mathcal{R}^{\prime}{ }_{c}$ represents the distance from the critical Reynolds number at which the bifurcation appears. The three parameters are reduced to two by defining a Landau time constant $\tau_{l}=\tau_{0} / \alpha$ and a final amplitude $A_{f}=A_{0} \sqrt{\alpha}$, such that the solution of Eq. (14) for $\alpha \geqslant 0$ becomes

$$
A(t)=\frac{A_{f} e^{\left(t / \tau_{l}\right)}}{\left[e^{\left(2 t / \tau_{l}\right)}+\left(A_{f} / A_{i}\right)^{2}-1\right]^{\frac{1}{2}}},
$$

where $A_{i}$ is the initial amplitude [36]. The energy in the wavy vortex near the instability is fitted to Eq. (15) with a leastsquares method, and the the fitted curve is plotted with the dashed gray line on the inset of Fig. 7. The coefficient of determination from the curve fit is $R^{2}=1-8.3 \times 10^{-4}$.

The speed at which the waves travel around the azimuth may be obtained by inspecting the spatiotemporal energy 


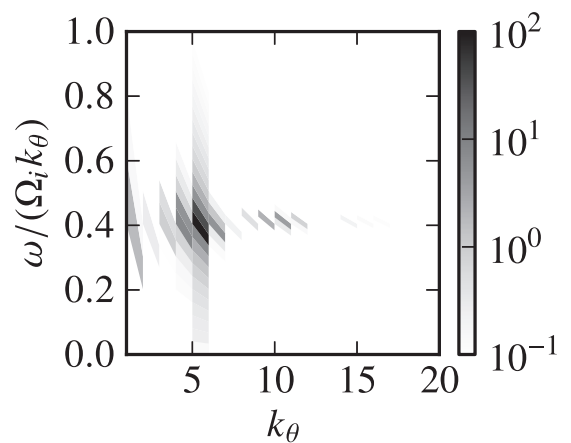

FIG. 8. Spatiotemporal energy spectra $E_{r}\left(k_{\theta}, \omega\right)$ for periodic boundary conditions in the axial $(z)$ direction. The vertical axes are plotted on $\omega / \Omega_{i} k_{\theta}$, which represents the traveling-wave phase speed [see Eq. (18)] of each Fourier mode in comparison with the rotation speed of the inner cylinder.

spectrum,

$$
E_{r}\left(k_{\theta}, \omega\right)=\frac{1}{L_{z}} \int_{0}^{L_{z}}\left|\hat{u}_{r}\left(k_{\theta}, z, \omega\right)\right|^{2} d z
$$

where

$$
\hat{u}_{r}\left(k_{\theta}, z, \omega\right)=\frac{1}{2 \pi L_{\tau}} \int_{0}^{2 \pi} \int_{\tau_{-}}^{\tau_{+}}\left\langle u_{r}\right\rangle_{r} e^{-i\left(k_{\theta} \theta+\omega \tau\right)} d \tau d \theta .
$$

The phase speed $v_{p}(k)$ of a traveling wave is given by

$$
v_{p}(k)=\frac{\varpi}{k},
$$

where $k$ and $\varpi(k)$ are the wave number and angular frequency of the wave. The angular frequencies $\varpi\left(k_{\theta}\right)$ are taken as the values of $\omega$ for which $E_{r}\left(k_{\theta}, \omega\right)$ is maximal. The maxima are observed in Fig. 8, where the frequencies have been normalized by the angular frequency of the inner cylinder $\Omega_{i}$. Alongside some weaker modes, the wavy vortex peak at $k_{\theta}=5$ is observed in the spectrum for the periodic-boundaries case. All $k_{\theta}$ modes have the same wave speed $\varpi / k_{\theta} \approx 0.4 \Omega_{i}=$ $d \varpi / d k_{\theta}=$ constant, which confirms that the waves travel around the azimuth with equal phase speeds, which are also equal to their group velocity.

\section{CONCLUSIONS}

The spontaneous bifurcation of cylindrical Couette flow and the formation of traveling-wavy vortices were simulated using molecular dynamics. Eight counter-rotating Taylor vortices were first formed in a cylindrical annulus with reflecting end wall boundary conditions. When the reflecting end walls were replaced with periodic boundary conditions, azimuthal waves formed and amplified along the vortices. The resulting wavyvortex flow field was consistent with previous experimental observations, and spectral analysis has confirmed that the growth of the wave amplitude is well described by Landau's theory for Hopf bifurcations. The results therefore demonstrate that molecular dynamics simulations can reproduce a bifurcation of this type without artificially exciting or imposing an initial macroscopic disturbance on the flow.

\section{APPENDIX: VOLUME AVERAGE STRESS TENSOR IN CYLINDRICAL COORDINATES}

An expression for the stress tensor, $\boldsymbol{\varsigma}$, averaged over a subvolume is sought in cylindrical coordinates. The present derivation starts from the expression for the stress at a point [37] and performs the averaging in the region of interest [38-40].

The kinetic $(K)$ and configurational $(U)$ parts of the stress tensor, local to a single point $\mathbf{x}$ in space, were expressed by Irving and Kirkwood [37] as

$$
\begin{aligned}
\boldsymbol{\zeta}^{(K)}(\mathbf{x} ; t) & =-\sum_{j=1}^{N} m\left\langle\left(\dot{\mathbf{x}}_{j}-\mathbf{v}\right)\left(\dot{\mathbf{x}}_{j}-\mathbf{v}\right) \delta\left(\mathbf{x}_{j}-\mathbf{x}\right) ; f\right\rangle, \\
\boldsymbol{\zeta}^{(U)}(\mathbf{x} ; t) & =\frac{1}{2} \sum_{j \neq k} \sum_{\neq=k}\left\langle\left(\nabla_{\mathbf{x}_{k}} \phi_{j k}\right) \mathbf{x}_{j k} O_{j k} \delta\left(\mathbf{x}_{j}-\mathbf{x}\right) ; f\right\rangle,
\end{aligned}
$$

where the inner product $\langle\alpha ; f\rangle$ defines the ensemble average of a quantity $\alpha$ in a system with phase space probability density function $f$. In the following, the angled bracket notation will be abandoned, although ensemble averaging remains implied. All particles have mass $m$, and their momenta and positions are given by $m \dot{\mathbf{x}}_{j}$ and $\mathbf{x}_{j}$, respectively, and the index $j$ spans all $N$ particles in the system. The peculiar velocity in the kinetic contribution to the stress is obtained by subtracting the streaming component $\mathbf{v}(\mathbf{x} ; t)$ from the particle velocity. The operator $O_{j k}$ acts on the Dirac $\delta$ function,

$$
O_{j k} \equiv\left[1-\frac{1}{2} \mathbf{x}_{j k} \cdot \nabla_{\mathbf{x}}+\cdots-\frac{1}{n !}\left(\mathbf{x}_{j k} \cdot \nabla_{\mathbf{x}}\right)^{n-1}+\cdots\right]
$$

The interaction potential between particles $j$ and $k$ is $\phi_{j k}$, and $\mathbf{x}_{j k}=\mathbf{x}_{k}-\mathbf{x}_{j}$. For pairwise interactions that depend on molecular separation only, the potential can be expressed as $\phi_{j k}=\phi\left(x_{j k}\right)$, where $x_{j k} \equiv\left|\mathbf{x}_{j k}\right|$. The force contribution from particle $k$ onto particle $j$ acts in the direction that is aligned with the shortest path between them. In Cartesian coordinates, the force is therefore

$$
\tilde{\mathbf{F}}_{j k} \equiv \nabla_{\tilde{\mathbf{q}}_{k}} \phi_{j k}=\frac{\tilde{\mathbf{q}}_{j k}}{\tilde{q}_{j k}} \frac{\partial \phi\left(\tilde{q}_{j k}\right)}{\partial \tilde{q}_{j k}} .
$$

The force-moment tensor may therefore be written in the familiar form,

$$
\left(\nabla_{\tilde{\mathbf{q}}_{k}} \phi_{j k}\right) \tilde{\mathbf{q}}_{j k}=\tilde{\mathbf{q}}_{j k} \tilde{\mathbf{F}}_{j k} .
$$

An expression of the force moment tensor in cylindrical coordinates, $\mathbf{M}_{j k}$, is obtained by first computing the tensor in Cartesian coordinates, and then rotating the result by the transformation matrix $\boldsymbol{\Theta}_{j}$,

$$
\mathbf{M}_{j k}=\boldsymbol{\Theta}_{j}\left(\tilde{\mathbf{q}}_{j k} \tilde{\mathbf{F}}_{j k}\right) \boldsymbol{\Theta}_{j}^{T} .
$$

Ultimately an evaluation of the stress in a region of interest, or equivalently the volume average of (A1) and (A2), is sought. One must first rewrite $O_{j k} \delta\left(\mathbf{x}_{j}-\mathbf{x}\right)$ in a more tractable form. First, consider the Taylor series expansion of 


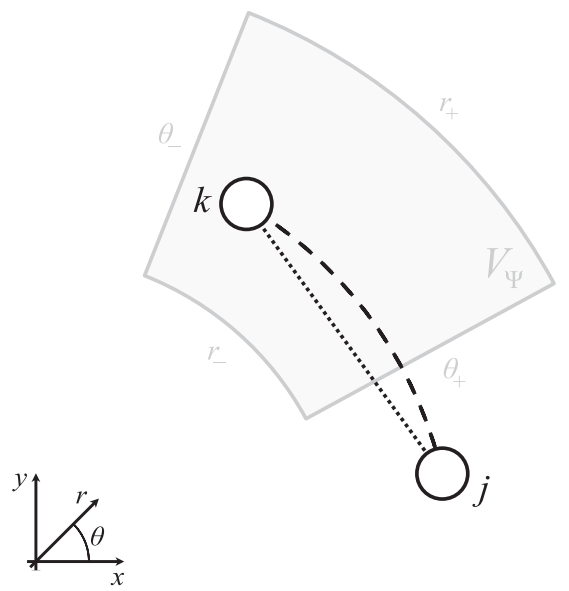

FIG. 9. Linear trajectories between two particles $j$ and $k$, in Cartesian coordinates (dotted line, showing $\tilde{\mathbf{q}}_{j}+s \tilde{\mathbf{q}}_{j k}$ ) and cylindrical polar coordinates (dashed line, showing $\mathbf{q}_{j}+s \mathbf{q}_{j k}$ ), as viewed along the $z$ axis. The volume of a cylindrical volume element in Eq. (A10), $V_{\Psi}$, is also plotted with its bounds in gray.

the Delta function,

$$
\begin{aligned}
\delta\left(\left(\mathbf{x}-\mathbf{x}_{j}\right)-s \mathbf{x}_{j k}\right)= & {\left[1-s \mathbf{x}_{j k} \cdot \nabla_{\mathbf{x}}+\cdots+\frac{s^{n-1}}{(n-1) !}\right.} \\
& \left.\times\left(-\mathbf{x}_{j k} \cdot \nabla_{\mathbf{x}}\right)^{n-1}+\cdots\right] \delta\left(\mathbf{x}-\mathbf{x}_{j}\right) .
\end{aligned}
$$

It is straightforward to show that the operation $O_{j k} \delta\left(\mathbf{x}_{j}-\mathbf{x}\right)$ is equivalent to

$$
O_{j k} \delta\left(\mathbf{x}_{j}-\mathbf{x}\right)=\int_{0}^{1} \delta\left(\mathbf{x}-\left(\mathbf{x}_{j}+s \mathbf{x}_{j k}\right)\right) d s,
$$

where $s \in[0,1]$ parameterizes the trajectory from $\mathbf{x}_{j}$ to $\mathbf{x}_{k}$ [41]. This trajectory can, however, be arbitrarily chosen and (A7) remains valid. Irving and Kirkwood [37] selected the line of centers connecting the two molecules, $\tilde{\mathbf{q}}_{j}+s \tilde{\mathbf{q}}_{j k}$. This is compared to the trajectory $\mathbf{q}_{j}+s \mathbf{q}_{j k}$ in cylindrical coordinates in Fig. 9. Irving and Kirkwood ultimately noted that this difference becomes negligible when the averaging volume is large relative to the molecular interaction range.

The average of $\boldsymbol{\zeta}^{(K)}$ and $\boldsymbol{\zeta}^{(U)}$ over a cylindrical element $\Psi$ is given by,

$$
\begin{aligned}
& \boldsymbol{S}_{\Psi}^{(K)}(t)=-\frac{1}{V_{\Psi}} \sum_{j=1}^{N} m \int_{V_{\Psi}}\left(\frac{\mathbf{p}_{j}}{m}-\mathbf{u}\right)\left(\frac{\mathbf{p}_{j}}{m}-\mathbf{u}\right) \delta\left(\mathbf{q}_{j}-\mathbf{q}\right) d V_{\Psi}, \\
& \boldsymbol{S}_{\Psi}^{(U)}(t)=\frac{1}{2 V_{\Psi}} \sum_{j \neq k} \sum_{0} \int_{0}^{1} d s \int_{V_{\Psi}} \mathbf{M}_{j k} \delta\left(\mathbf{q}-\left(\mathbf{q}_{j}+s \mathbf{q}_{j k}\right)\right) d V_{\Psi} .
\end{aligned}
$$

In the above expression, $\mathbf{v}(\mathbf{x} ; t)$ has been replaced by $\mathbf{u}(\mathbf{q} ; t)$, and the volume of the cylindrical element,

$$
V_{\Psi}=\frac{1}{2}\left(r_{+}^{2}-r_{-}^{2}\right)\left(\theta_{+}-\theta_{-}\right)\left(z_{+}-z_{-}\right),
$$

is shown in Fig. 9.

The volume averages in Eqs. (A8) and (A9) yield the following expressions for the kinetic and configurational components of the stress tensor,

$$
\begin{gathered}
\boldsymbol{S}_{\Psi}^{(K)}(t)=-\frac{1}{V_{\Psi}} \sum_{j=1}^{N} m\left(\frac{\mathbf{p}_{j}}{m}-\mathbf{u}\right)\left(\frac{\mathbf{p}_{j}}{m}-\mathbf{u}\right) \Lambda_{j}, \\
\boldsymbol{S}_{\Psi}^{(U)}(t)=\frac{1}{2 V_{\Psi}} \sum_{j \neq k} \sum_{j k} \mathbf{M}_{j k} .
\end{gathered}
$$

In the above, $\Lambda_{j}$ is unity if particle $j$ lies within the averaging volume and zero otherwise, and $l_{j k}$ is the fraction of the line connecting particles $j$ and $k$ that lies within the averaging volume.
[1] J. Koplik, J. R. Banavar, and J. F. Willemsen, Phys. Rev. Lett. 60, 1282 (1988).

[2] D. J. Evans and Peter T. Cummings, Ind. Eng. Chem. Res. 31, 1237 (1992).

[3] A. Vadakkepatt, Y. Dong, S. Lichter, and A. Martini, Phys. Rev. E 84, 066311 (2011).

[4] A. Morozov and W. van Saarloos, Phys. Rep. 447, 112 (2007).

[5] P. Luchini, in 61st Annual Meeting of the APS Division of Fluid Dynamics (APS (AIP), New York, 2008), Vol. 53.

[6] D. C. Rapaport, Phys. Rev. Lett. 60, 2480 (1988).

[7] A. Puhl, M. M. Mansour, and M. Mareschal, Phys. Rev. A 40, 1999 (1989).

[8] D. C. Rapaport and E. Clementi, Phys. Rev. Lett. 57, 695 (1986).

[9] S. T. Cui and D. J. Evans, Mol. Simul. 9, 179 (1992).

[10] D. Hirshfeld and D. C. Rapaport, Phys. Rev. Lett. 80, 5337 (1998).

[11] D. Hirshfeld and D. C. Rapaport, Phys. Rev. E 61, R21(R) (2000).

[12] D. C. Rapaport, J. Phys.: Condens. Matter 26, 503104 (2014).
[13] G. P. King, W. Lee, Y. Li, H. L. Swinney, and P. S. Marcus, J. Fluid Mech. 141, 365 (1984).

[14] A. Akonur and R. M. Lueptow, Phys. Fluids 15, 947 (2003).

[15] G. I. Taylor, Philos. Trans. R. Soc., A 223, 289 (1923).

[16] D. Coles, J. Fluid Mech. 21, 385 (1964).

[17] K. W. Schwarz, B. E. Springett, and R. J. Donnelly, J. Fluid Mech. 20, 281 (1964).

[18] J. A. Cole, J. Fluid Mech. 75, 1 (1976).

[19] C. D. Andereck, S. S. Liu, and H. L. Swinney, J. Fluid Mech. 164, 155 (1986).

[20] S. Chandrasekhar, Hydrodynamic and Hydromagnetic Stability, Dover Books on Physics Series (Dover Publications, New York, 1961).

[21] A. Davey, R. C. Di Prima, and J. T. Stuart, J. Fluid Mech. 31, 17 (1968).

[22] M. Nagata, J. Fluid Mech. 188, 585 (1988).

[23] R. C. Di Prima and H. L. Swinney, in Hydrodynamic Instabilities and the Transition to Turbulence (Springer, Berlin, Germany, 1985), pp. 139-180. 
[24] P. S. Marcus, J. Fluid Mech. 146, 45 (1984).

[25] P. S. Marcus, J. Fluid Mech. 146, 65 (1984).

[26] C. A. Jones, J. Fluid Mech. 157, 135 (1985).

[27] J. Abshagen, O. Meincke, G. Pfister, K. A. Cliffe, and T. Mullin, J. Fluid Mech. 476, 335 (2003).

[28] S. Dong, J. Fluid Mech. 587, 373 (2007).

[29] S. Stefanov and C. Cercignani, J. Fluid Mech. 256, 199 (1993).

[30] D. Riechelmann and K. Nanbu, Phys. Fluids A 5, 2585 (1993).

[31] D. Riechelmann and K. Nanbu, Phys. Fluids 9, 811 (1997).

[32] L. D. Landau and E. M. Lifshitz, Fluid Mechanics, 2nd ed., Course of Theoretical Physics (Pergamon Press, Oxford, 1966).
[33] M. P. Allen and D. J. Tildesley, Computer Simulation of Liquids (Clarendon Press, Oxford, 1987).

[34] J. Petravic and P. Harrowell, J. Chem. Phys. 124, 014103 (2006).

[35] D. M. Heyes, Phys. Rev. B 37, 5677 (1988).

[36] J. Abshagen and G. Pfister, Phys. Rev. E 78, 046206 (2008).

[37] J. H. Irving and J. G. Kirkwood, J. Chem. Phys. 18, 817 (1950).

[38] D. M. Heyes, E. R. Smith, D. Dini, and T. A. Zaki, J. Chem. Phys. 140, 054506 (2014).

[39] E. R. Smith, D. M. Heyes, D. Dini, and T. A. Zaki, Phys. Rev. E 85, 056705 (2012).

[40] D. M. Heyes, E. R. Smith, D. Dini, and T. A. Zaki, J. Chem. Phys. 135, 024512 (2011).

[41] M. Han and J. S. Lee, Phys. Rev. E 70, 061205 (2004). 